\title{
Nivel de depresión entre estudiantes de Enfermería y Nutrición en una universidad pública de Aguascalientes
}

\author{
Pineda-Pedraza Miguel Ángel*, González-Flores Silvia Patricia**
}

\section{Resumen \\ - Introducción. La depresión es un trastorno mental universal, \\ - cuya principal perturbación es la alteración del humor o del \\ - afecto. Según estudios previos, el promedio de la prevalencia \\ - de depresión en los estudiantes es del 13.5\% con una ten- \\ - dencia a la disminución en los últimos semestres en compa- \\ - ración con el primer año. Objetivo: Determinar la prevalencia \\ - de depresión entre los estudiantes de las carreras de nutri- \\ - ción y enfermería. Metodología: Estudio transversal y compa- \\ - rativo, para la recolección de datos se usó la escala de Zung \\ - con nivel de confiabilidad de 0.88 por alfa de Cronbach en \\ - una muestra de 37I estudiantes de la Universidad Autó- \\ - noma de Aguascalientes (UAA) durante marzo del 2016, \\ - mediante un muestreo estratificado simple. Resultados: La \\ - edad promedio fue de 20 años, correspondió al sexo feme- \\ - nino el $72.8 \%$, y el $27.2 \%$ al sexo masculino. El promedio \\ - de la prevalencia de depresión en el total de estudiantes \\ - evaluados fue $49.5 \%$. No se encontró diferencia significativa \\ - al comparar las medias del nivel de depresión de ambas \\ - carreras ( $p=0.677)$. Conclusión: Los resultados obtenidos \\ - nos demuestran que el trastorno depresivo es uniforme en \\ - ambas carreras. Los alumnos de las carreras de enfermería \\ - y nutrición de la UAA muestran índices depresivos leves en \\ - casi la mitad de los estudiantes evaluados. LUXMÉDICA AÑ011, \\ NÚMERO 34, SEPTIEMBRE-DICIEMBRE 2016, PP 53-62}

$\bullet$

Palabras clave: Nivel de depresión, estudiantes universitarios, Zung.

\section{Abstract}

Introduction. Depression is a universal mental disorder, whose main disturbance is an alteration of humor or affection. According to previous studies, the average prevalence of depression in students is of $13.5 \%$ with a downward trend in the last semesters in comparison with the first year. Objective: To determine the prevalence of depression in students of nutrition and nursing. Methodology: cross-sectional and comparative study for the collection of data was used the Zung scale with level of reliability of 0.88 by Cronbach's Alpha in a sample of 371 students of the University of Aguascalientes (UAA) during March 2016, using a simple stratified sampling. Results: the mean age was 20 years, corresponded to the females the $72.8 \%$ and $27.2 \%$ males. The prevalence of depression in the total of evaluated students averaged 49.5\%. No significant difference was found to compare the means of the level of depression from both races $(p=0.677)$. Conclusion: the results obtained show that the disorder depressive is uniform in both disciplines. Nursing and Nutrition students of UAA show mild depressive indexes in almost half of tested students. LUXMÉDICA AÑ011, NÚMERO 34, SEPTIEMBRE-DICIEMBRE 2016, PP 53-62

Estudiante de la Licenciatura de Enfermería del Centro de Ciencias de la Salud de la Universidad Autónoma de Aguascalientes, Aguascalientes, México.

** Profesora investigadora de tiempo completo, Departamento de Enfermería, Centro de Ciencias de la Salud, Universidad Autónoma de Aguascalientes, Aguascalientes, México.

Fecha de recibido: 24 de mayo 2016

Fecha de aceptado: 13 de agosto 2016

Correspondencia: MCBE Silvia Patricia González Flores, Departamento de Enfermería del Centro de Ciencias de la Salud de la Universidad Autónoma de Aguascalientes. Av. Universidad No 940, Edificio 105 Cd. Universitaria. Código postal. 20131. México. Teléfono. 014499108437. Aguascalientes, Ags. Correo electrónico: spgonza@correo.uaa.mx 


\section{Introducción}

La depresión es un trastorno mental universal, cuya principal perturbación es la alteración del humor o del afecto. La alteración del humor tiene repercusión en el nivel global de actividades de la persona afectada. ${ }^{1}$ De acuerdo con los resultados de la Organización Mundial de la Salud (OMS), la depresión es un problema de salud a nivel mundial que afecta a toda la población en general. En el mundo hay más de 350 millones de personas con depresión, y tiene una distribución cosmopolita. ${ }^{2}$

La depresión en México no es una moda, constituye un severo problema de salud pública que afecta entre $12 \%$ y $20 \%$ a personas adultas, es decir, entre 18 y 60 años. La depresión es una alteración patológica del estado de ánimo con descenso del humor en el que predominan los síntomas afectivos (sentimientos de dolor profundo, de culpa, de soledad, tristeza patológica, decaimiento, irritabilidad, desesperanza, sensación subjetiva de malestar e impotencia frente a las exigencias de la vida); además, en mayor o menor grado, están presentes síntomas de tipo cognitivo (baja atención, concentración y memoria, pensamientos de muerte o ideación suicida), volitivo (apatía, anhedonia, retardo psicomotor, descuido en sus labores cotidianas) y somático (cefalea, fatiga, dolores, alteraciones del sueño, somatizaciones, propensión a infecciones, etc.), por lo que es una afectación global de la vida psíquica. ${ }^{3}$ Existen estudios que encontraron mayor riesgo en personas con antecedentes familiares de trastornos afectivos, con experiencias infantiles: muerte parental temprana, medio familiar caótico, maltrato físico y psicológico, con eventos vitales negativos tales como ausencia de confidente, pobre apoyo social y ambiente disruptivo. ${ }^{4,5}$

La depresión se considera uno de los desórdenes de la salud mental de mayor registro en los centros de salud de primer nivel de atención. Asimismo, dentro de la población universitaria, este trastorno es uno de los principales motivos de consulta atendidos a través de los servicios de salud universitarios. ${ }^{6}$

La etapa del ciclo vital en la que se encuentran la mayoría de los estudiantes universitarios de pregrado, es la adolescencia tardía. Y es justamente allí, cuando se acentúan algunos problemas de salud mental que no se presentan con tanta frecuencia en etapas previas, como los trastornos del estado de ánimo y la ansiedad. Sumado a lo anterior, las mayores exigencias académicas presentes en la educación superior, son reconocidas por los estudiantes como uno de los factores que generan mayores niveles de estrés. ${ }^{7}$

Una de las teorías más significativas sobre la depresión, es la teoría cognitivo-conductual de Beck, que interpreta la depresión como una consecuencia de las distorsiones que una persona puede hacer al procesar la información que obtiene de lo que le rodea. Según esta teoría, las personas poseen en su pasado una serie de experiencias negativas que detonan un esquema vital pesimista en tres sentidos: 
se crean un concepto negativo de sí mismas, de lo que las rodean y de sus futuros. ${ }^{8}$

Estudiar una carrera de ciencias de la salud se caracteriza por una exigencia académica elevada. ${ }^{9}$ La prevalencia de depresión reportada por múltiples estudios realizados en población universitaria oscila entre el $25 \%$ y el $50 \%$, y ello está determinado, en parte, por los diferentes instrumentos utilizados para su medición. ${ }^{10}$

Feldman, L. y colaboradores han evaluado la relación que existe de la depresión con aspectos ambientales tales como las condiciones físicas de la vivienda; encontraron que existe un leve aumento de la depresión cuando la persona consideraba que su espacio físico era desfavorable. Otros elementos como nivel de escolaridad, y la poca satisfacción con los logros alcanzados, se percibían desfavorablemente en la medida en que aumentaba la gravedad de la depresión. ${ }^{11}$

Diversos autores han mostrado preocupación por la salud mental de los estudiantes universitarios, que explican la prevalencia de variables individuales relacionadas con la depresión en los estudiantes universitarios, tales como los antecedentes familiares y personales de depresión, dificultades académicas, inestabilidad económica, diagnóstico de una enfermedad grave, muerte de un ser querido, separación de los padres, entre otros. La prevalencia de depresión en universitarios que inician el año académico es mayor en la etapa final de la carrera y puede ser atribuible, entre otros factores, a un fenómeno acumulativo de carga académica que actualmente genera este proceso educativo. Los antecedentes personales para depresión y los factores situacionales se asocian a la presencia de depresión de una forma diferente de acuerdo al año académico. ${ }^{12}$

En el ámbito universitario uno de los principales factores asociados a la depresión es el estrés académico, que se constituye en un elemento detonante y que perpetúa la enfermedad. Fisher y Hood demostraron que los estudiantes experimentan un incremento significativo en los niveles de depresión, síntomas obsesivos y pérdida de concentración tras seis semanas de permanencia en la universidad. Este medio académico demanda desempeños sobresalientes para alcanzar el éxito, y dicha exigencia implica para el ser humano un proceso de adaptación que puede resultar en la generación de estrés. ${ }^{13}$

Las dificultades en las relaciones con los docentes (manejo de autoridad, estilo docente, dificultad en la intercomunicación, inequidad en la evaluación, sobrecarga de asignaturas, horarios, metodología del docente y deficiencia en los métodos de estudio del alumno) pueden conducir a la depresión. En un estudio sobre depresión en estudiantes de medicina la prevalencia global de depresión fue de $21.6 \%$, siendo casi de dos mujeres por un hombre. ${ }^{14}$ Miranda Bastidas, Carlos Alberto, Joffre Velázquez son autores que han mostrado interés por la salud mental de los estudiantes universitarios, argumentando que el conjunto de las actividades académicas puede influir en su bienestar físico y psicológico. El ingreso a la universidad constituye un cambio muy importante para los jóvenes, tanto en términos sociales como académicos. ${ }^{15,16}$

Czernik y cols. mencionan que el sentirse bajo presión por los exámenes, experimentar presión por parte de sus profesores y tener dificultades económicas y relacionales, son características que se ha demostrado que están asociadas con una sintomatología depresiva y ansiosa en estudiantes de ciencias de la salud. ${ }^{17}$ Por otro lado, Jou $\mathrm{H}$. plantea que la falta de apoyo social, familiar y, nuevamente, los altos niveles de estrés, pueden devenir en depresión. ${ }^{18}$

En otro estudio se reporta que la mayor exigencia académica está asociada con mayores niveles de estrés, considerado como un factor determinante para depresión. Su 
estudio realizado en estudiantes de carreras relacionadas con la salud como medicina, odontología, enfermería y psicología mostró un nivel elevado de prevalencia de estrés académico (36,3\%), ${ }^{19}$ mientras que los hallazgos de Stewart y colaboradores, señalan que la depresión se asocia más a experiencias o sentimientos de fracaso académico. ${ }^{20}$

En México, de acuerdo a la Encuesta $\mathrm{Na}$ cional de Epidemiología Psiquiátrica que se realizó entre los años 2001-2002, en lo referente al episodio depresivo mayor, en particular, se documentó una prevalencia en la población total del orden del 3.3\% alguna vez en la vida y del $1.5 \%$ durante los 12 meses previos a la encuesta. ${ }^{21}$

Gutiérrez Rodas en su estudio encontró que la prevalencia de la depresión en estudiantes universitarios es similar a lo reportado a nivel mundial, utilizando la escala de Zung encontró una prevalencia de 47,3 \%, donde la depresión leve fue la más frecuente $(29,9 \%)$. De la misma forma se encontró una mayor prevalencia en el género femenino. También encontró que la prevalencia de depresión en estudiantes universitarios fue mayor para las mujeres, aunque la diferencia en los casos de la depresión severa no fue estadísticamente significativa y estas diferencias dadas entre hombres y mujeres han sido explicadas desde múltiples visiones que han permitido establecer cómo la estructura psicobiológica y algunos factores psicosociales hacen más vulnerable al género femenino frente a estos trastornos. ${ }^{22}$

Riveros refiere una investigación realizada en Lima, Perú, con 500 estudiantes, en la cual el $26 \%$ presentó alguna forma de depresión. ${ }^{23}$ Por su parte, Cantoral, Méndez y Nazar en México, encontraron que la prevalencia de sintomatología compatible con depresión entre los estudiantes fue del $62,7 \%$. ${ }^{24}$ Riveros también menciona que la prevalencia de la depresión en la población estudiantil universitaria es cambiante y puede variar de acuerdo con el objeto de estudio, del nivel socio-cultural y del ámbito geográfico.
Valencia $M$. documentó que el 85,2\% de la población encuestada perteneciente al programa educacional de enfermería de la Universidad Libre de Pereira presentaba algún tipo de depresión, sin ser la depresión grave o severa la de mayor prevalencia. ${ }^{25}$ Terrones (2014) observó que "en todas las carreras del Centro de Ciencias de la Salud hay una tendencia al incremento de la prevalencia de depresión en los últimos semestres en comparación con el primer año". Terrones y cols encontraron que la prevalencia de depresión en los estudiantes evaluado fue del $13.5 \%$, predominando el nivel de depresión leve en todos los casos en los que se presentaron síntomas. Al desglosar las frecuencias de depresión del primero y último año de cada carrera, encontró que para Enfermería fue de $12.5 \%$ y $19.1 \%$, y para Nutrición, $5 \%$ y $40.6 \%$, respectivamente. ${ }^{26}$

Sidana Surbhi (2012) afirma que la prevalencia de depresión en estudiantes de carreras pertenecientes al centro de ciencias de la salud del primer año, cuando los comparan con alumnos del último año de la carrera, presentan un menor nivel de depresión durante el último semestre de la carrera. ${ }^{27}$ De acuerdo al estudio de Ferreira, el 19,2\% de los estudiantes de enfermería presentan depresión; siendo que $12,5 \%$ presentaban disforia, 6,3\% depresión moderada y 0,4\% presentaba depresión grave. ${ }^{28}$

En los últimos años, la depresión se encuentra dentro de los problemas que más causa sufrimiento a la población estudiantil y que además se cataloga como uno de los principales problemas de salud en actualidad a nivel estatal como nacional. Sin diferencia de edad, sexo, raza, nivel socioeconómico, la convierte en uno de los motivos más comunes de consulta clínica. ${ }^{29}$

El presente estudio pretende determinar el nivel de depresión entre los estudiantes de las carreras de nutrición y enfermería de una universidad pública de Aguascalientes. 


\section{Material y métodos}

Se realizó un estudio comparativo, transversal, y prospectivo. La recolección de datos se llevó a cabo en marzo de 2016 en la Universidad Autónoma de Aguascalientes. El número de estudiantes inscritos en las carreras de Enfermería y de Nutrición fue de 722 alummos. El muestreo fue por conveniencia. Fueron evaluados 371 estudiantes universitarios: de enfermería fueron incluidos 231 estudiantes y de nutrición 140 estudiantes. Quedaron incluidos estudiantes de todos los semestres que aceptaron participar y que se encontraban en ese momento en el salón de clases. El instrumento fue aplicado en el periodo entre una clase y otra; el tiempo promedio para la contestación del cuestionario fue de ocho a diez minutos. Se excluyeron a los estudiantes que no desearon participar en el estudio, a los que no se encontraban en el salón de clases al momento de la aplicación y aquellos estudiantes que se les había aplicado ya el instrumento anteriormente.
El instrumento utilizado fue la escala de autoevaluación para la depresión de Zung (SDS); cuenta con una confiabilidad de la medida se obtuvo un alfa de Cronbach global de 0.887. El instrumento de depresión de Zung evalúa algunos de los síntomas de la depresión. El cuestionario consta de 20 afirmaciones, a las que se les da un puntaje que va de 1 a 4 dependiendo de la respuesta: 1 para la respuesta nunca o muy pocas veces; 2 para algunas veces; 3 para frecuentemente; y 4 para siempre. En las preguntas 2, 5, 6, 11, 12, 14, 16, 17, 18 , y 20 se invierte el orden del puntaje. El puntaje para evaluar la severidad de los síntomas fueron los siguientes: de 0 hasta 49 puntos, sin síntomas; más de 50 puntos, depresión leve o mínima; más de 60 puntos, depresión moderada y más de 70 puntos, depresión severa.

La depresión se midió en rangos: sin síntomas de 0-49 puntos; de 50-59 puntos, depresión leve o mínima; de 60-69 puntos, depresión moderada y más de 70 puntos, depresión severa.

| | | | | | | | | | | | | | | | | | | | | | | | | | | | | | | | | | | | | | | | | | | | | | | | | | | | | | | | | | | | | | | | | | | | | | | | | | | | | | | | | | | | | | | | | | | | | | | | | | | | | | | | | | | | |

\section{Resultados}

Fueron estudiados 371 estudiantes universitarios del área de la salud, de la licenciatura en enfermería (231 estudiantes) y de la licenciatura en nutrición (140 estudian- tes); con una edad promedio de 20 años; el $72.77 \%$ correspondió al sexo femenino y tan sólo el $27.22 \%$ al sexo masculino; como se muestra en la tabla 1.

\section{Tabla}

Distribución de la población estudiada según sexo y carrera a la que pertenece

\begin{tabular}{|llllllllc|}
\hline & \multicolumn{9}{c}{ SEXO DEL PARTICIPANTE } & Total \\
\hline & & MASCULINO & \multicolumn{1}{c|}{ FEMENINO } & & \\
\hline CARRERA QUE CURSA & ENFERMERÍA & 70 & $18.86 \%$ & 161 & $43.39 \%$ & 231 & $62.26 \%$ \\
\hline & NUTRICIÓN & 31 & $8.35 \%$ & 109 & $29.38 \%$ & 140 & $37.73 \%$ \\
\hline Total & & 101 & $27.22 \%$ & 270 & $72.77 \%$ & 371 & $100 \%$ \\
\hline
\end{tabular}

FUENTE: Nivel de depresión entre estudiantes de Enfermería y Nutrición de una Universidad Pública de Aguascalientes. Escala de Autoevaluación de Zung. 
De acuerdo a la distribución por semestre de los estudiantes evaluados de ambas carreras se obtuvo lo que observamos en la tabla 2.

\section{Tabla 2}

\section{Total de alumnos estudiados por semestre}

\begin{tabular}{|lc|c|c|c|c|c|ccc|}
\hline & \multicolumn{1}{c}{ SEMESTRE } & \multicolumn{1}{c}{ Total } \\
\hline CARRERA & 1RO & 2DO & 3ER & 4TO & $5 T O$ & $6 T O$ & $7 M O$ & 8 VO \\
\hline ENFERMERÍA & 24 & 38 & 18 & 42 & 18 & 37 & 19 & 35 & 231 \\
\hline NUTRICIÓN & 19 & 15 & 17 & 17 & 18 & 18 & 17 & 19 & 140 \\
\hline Total & 43 & 53 & 35 & 59 & 36 & 55 & 36 & 54 & 371 \\
\hline
\end{tabular}

FUENTE: Nivel de depresión entre estudiantes de Enfermería y Nutrición de una Universidad Pública de Aguascalientes. Escala de Autoevaluación de Zung.

La prevalencia de depresión en el total la licenciatura en nutrición y el $23.96 \%$ los de estudiantes evaluados fue de $49.5 \%$; estudiantes de la licenciatura en enfermeocupando el $25.54 \%$ los estudiantes de ría (figura 1).

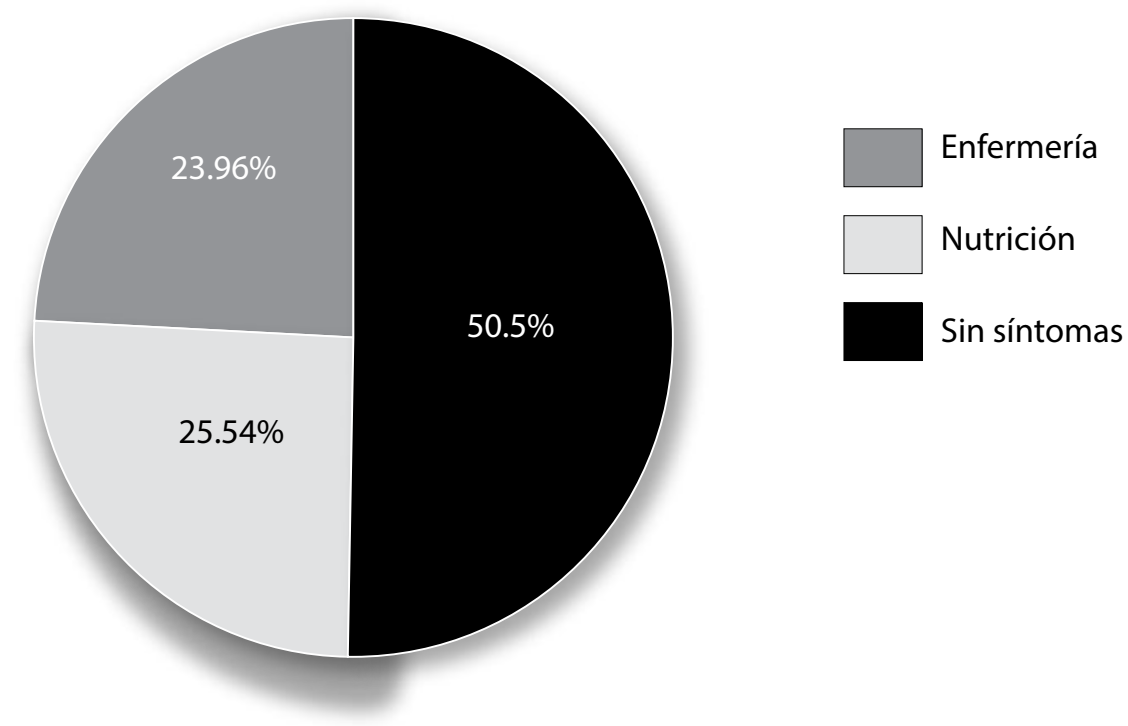

Figura 1. Distribución de la frecuencia de la depresión en los estudiantes evaluados

La prevalencia de depresión en la ca- encontrar ningún caso de depresión severa tegoría ligeramente deprimido en el total y el resto no presentó síntomas de deprede estudiantes evaluados fue de $48.26 \%$, sión (figura 2).

moderadamente deprimidos el $1.24 \%$, sin 


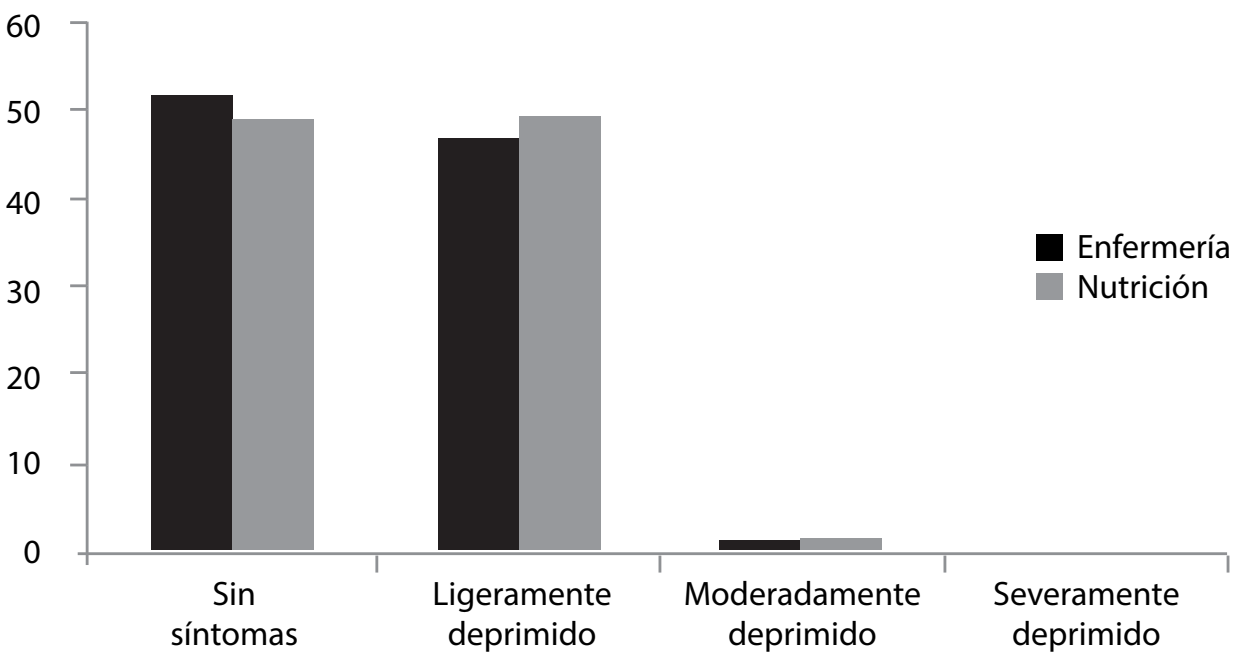

Figura 2. Distribución de la prevalencia de depresión de acuerdo a su severidad y a la licenciatura que pertenecen

La prevalencia de depresión en el total de estudiantes evaluados de la licenciatura de nutrición fue de 50.7\%; ocupa mayor porcentaje la categoría de depresión leve.
En los semestres tercero y sexto se encontró mayor frecuencia de depresión para esta licenciatura, como se puede observar en la tabla 3.

\section{Tabla 3}

Desglose de la frecuencia de depresión de acuerdo al semestre que se cursa en la licenciatura de Nutrición

\begin{tabular}{|lccccc|}
\hline \multicolumn{7}{c|}{ Nivel de depresión } \\
\hline Semestre que cursa & Sin síntomas & Leve & Moderada & Severa & TOTAL \\
\hline Primero & $7.8 \%$ & $5.7 \%$ & $0 \%$ & $0 \%$ & $13.6 \%$ \\
\hline Segundo & $3.5 \%$ & $7.1 \%$ & $0 \%$ & $0 \%$ & $10.7 \%$ \\
\hline Tercero & $4.2 \%$ & $7.8 \%$ & $0 \%$ & $0 \%$ & $12.1 \%$ \\
\hline Cuarto & $5 \%$ & $6.4 \%$ & $0.7 \%$ & $0 \%$ & $12.1 \%$ \\
\hline Quinto & $6.4 \%$ & $6.4 \%$ & $0 \%$ & $0 \%$ & $12.9 \%$ \\
\hline Sexto & $5 \%$ & $7.1 \%$ & $0.7 \%$ & $0 \%$ & $12.9 \%$ \\
\hline Séptimo & $7.8 \%$ & $4.2 \%$ & $0 \%$ & $0 \%$ & $12.1 \%$ \\
\hline Octavo & $9.3 \%$ & $4.2 \%$ & $0 \%$ & $0 \%$ & $13.6 \%$ \\
\hline TOTAL & $\mathbf{4 9 . 3 \%}$ & $\mathbf{4 9 . 3 \%}$ & $\mathbf{1 . 4} \%$ & $\mathbf{0} \%$ & $\mathbf{1 0 0} \%$ \\
\hline
\end{tabular}

La prevalencia de depresión en el total de estudiantes evaluados de la licenciatura de enfermería fue de $48.4 \%$; ocupa, igual que en el caso de los estudiantes de nutrición mayor porcentaje la categoría de depresión leve. El cuarto semestre fue donde hubo mayor nivel de depresión para esta licenciatura, como se puede observar en la tabla 4. 


\section{Tabla 4}

Desglose de la frecuencia de depresión de acuerdo al semestre que se cursa en la licenciatura de Enfermería

\begin{tabular}{|lccccc|}
\hline \multicolumn{7}{c|}{ Nivel de depresión } \\
\hline Semestre que cursa & Sin síntomas & Leve & Moderada & Severa & TOTAL \\
\hline Primero & $5.2 \%$ & $5.2 \%$ & $0 \%$ & $0 \%$ & $10.4 \%$ \\
\hline Segundo & $13 \%$ & $3.5 \%$ & $0 \%$ & $0 \%$ & $16.4 \%$ \\
\hline Tercero & $5.6 \%$ & $2.2 \%$ & $0 \%$ & $0 \%$ & $7.8 \%$ \\
\hline Cuarto & $8.7 \%$ & $9 \%$ & $0.4 \%$ & $0 \%$ & $18.2 \%$ \\
\hline Quinto & $3 \%$ & $4.8 \%$ & $0 \%$ & $0 \%$ & $7.8 \%$ \\
\hline Sexto & $7.4 \%$ & $8.2 \%$ & $0.4 \%$ & $0 \%$ & $16 \%$ \\
\hline Séptimo & $1.7 \%$ & $6 \%$ & $0.4 \%$ & $0 \%$ & $8.2 \%$ \\
\hline Octavo & $6.9 \%$ & $8.2 \%$ & $0 \%$ & $0 \%$ & $15.2 \%$ \\
\hline TOTAL & $\mathbf{5 1 . 5 \%}$ & $\mathbf{4 7 . 2} \%$ & $\mathbf{1 . 2} \%$ & $\mathbf{0} \%$ & $\mathbf{1 0 0} \%$ \\
\hline
\end{tabular}

En estas mismas tablas, también observamos que no existe una tendencia ni al incremento o disminución de las frecuencias en relación con el semestre cursado. Al comparar el promedio de depresión entre las carreras no se obtuvo diferencia significativa $(p=0.667)$.

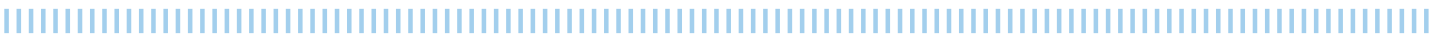

\section{Discusión}

El promedio de prevalencia en los estudiantes universitarios fue similar al que Méndez y $\mathrm{Na}$ zar encontraron en su estudio realizado también realizado a estudiantes universitarios. ${ }^{22}$

El presente estudio no pudo confirmar lo que Riveros comenta acerca de que la prevalencia de la depresión en la población estudiantil universitaria puede variar de acuerdo con el objeto de estudio, del nivel socio-cultural y del ámbito geográfico ya que el instrumento que se utilizó en este estudio no mide dichas variables sociodemográficas. ${ }^{21}$ Comparando nuestros resultados con los obtenidos por el grupo de Valencia y cols, encontramos que la prevalencia en este estudio es menor a la referida por ellos, donde identificaron que el $85,2 \%$ de los estudiantes de enfermería evaluados, presentaron algún tipo de depresión, sin ser la depresión grave o severa la de mayor prevalencia. ${ }^{23}$
En otro estudio, realizado por Terrones y cols, encontraron que el promedio de la prevalencia de depresión en los estudiantes del área de la salud, correspondió al $13.5 \%$ y observaron una tendencia al incremento de la prevalencia de depresión en los últimos semestres en comparación con el primer año, ${ }^{24}$ mientras que Sidana Surbhi afirma que hay un decremento los últimos semestres en comparación con el primer año. ${ }^{25}$ El presente estudio muestra una prevalencia mayor $(49.5 \%)$ que la reportada con anterioridad (13.5\%). El estudio también difiere de opinión tanto con Terrones como con Surbhi debido a que en los resultados se observa que no existe un orden descendente ni tampoco ascendente en cuanto al nivel de depresión en conforme se avanza al siguiente semestre. En este estudio, el semestre de la licenciatura de enfermería en el que se registró mayor prevalencia de estudiantes con depresión 
fue cuarto semestre; para la licenciatura de nutrición los semestres con mayor prevalencia de depresión, fueron el tercero y el cuarto. Con base en los resultados lo único en lo que se concuerda con Terrones, es que la categoría de depresión leve es la que tiene el mayor porcentaje de los casos. En otro estudio realizado en una universidad privada de Lima, Perú, se encontró en su centro de ciencias de la salud que los estudiantes de género femenino de las carreras de enfermería y medicina tenían los índices de depresión más altos de todas las demás carreras, incluyendo la de nutrición.

Es probable que los estudiantes de estas carreras, de nutrición y de enfermería, que en su mayoría son mujeres, se encuentren en situaciones de mayor estrés tengan que cumplir exigencias y expectativas que les solicitan sus carreras; claro que esto se puede también deber a un sesgo porque el instrumento de Zung tiene mayor peso para los síntomas de ansiedad que la sintomatología afectiva depresiva.

\section{Conclusiones}

Encontramos que la prevalencia de depresión entre los estudiantes evaluados de las licenciaturas de nutrición y de enfermería es similar. Cabe resaltar que los alumnos de las carreras de enfermería y nutrición de la UAA muestran índices de depresión leve en casi la mitad de la muestra. Es aceptado que la depresión en los estudiantes universitarios puede afectar de manera negativa en su rendimiento académico, lo cual puede generar más problemas adyacentes a este trastorno depresivo.

Debido a esto se recomienda fomentar en esta institución: la creación de planes de intervenciones dirigidos hacia prevenir y detectar, y en su caso, disminuir la incidencia de estudiantes con depresión.

También se pretende que las autoridades correspondientes analicen los programas de estudio y cargas académicas, de los semestres en los que se presentan mayor número de casos de depresión por parte de los estudiantes.

Se propone canalizar al alumno en caso de detectar depresión moderada o severa como estrategia que se espera que las autoridades institucionales educativas implementen.

\section{Bibliografía}

1. Marcelo Pio de Almeida. Trastornos depresivos. Revista de Psiquiatria do Rio Grande do Sul - SPRS. São Paulo, 2005; 10(2): 1-4.

2. WHO (OMS) Mental Health and development. Targeting people with mental health conditions as a vulnerable group. WHO Library Cataloguing-in Publication Data. 2010. Disponible en http://apps.who.int/iris/bitstr am/10665/44257/1/9789241563949_eng.pdf

3. Guía de Práctica Clínica. Diagnóstico y tratamiento del trastorno depresivo en el adulto. Disponible en http://www.cenetec.salud.gob.mx/descargas/gpc/ catalogomaestro/161_gpc_trastorno_depresivo/ imss_161er.pdf
4. Vieta, Eduard M; Benabarre, Antoni; ColGastó, Cristòbal Gastó Kéller. Suicidal Behavior in Bipolar I and Bipolar II Disorder. Revista Nervous \& Mental Disease,1997; 185(6): 407-409.

5. Marcela Guzmán Sánchez, Rodrigo Andrés Mardones Carrasco, Marcela Alejandra Romero Carvajal. La depresión en niños y adolescentes en Chile: apuntes para una psicoterapia de la depresión infantil desde un enfoque constructivista-evolutivo. Revista virtual SEPYPNA, 2011; 45(2): 52-52

6. Gómez-Restrepo C, Ospina MB, Díaz Granados N. Carga de la enfermedad mental en el mundo: un esbozo de la relevancia de los trastornos psiquiátricos. Revista Panam Salud Publica online, 2004; 16(6): 386-378. 
7. Félix Cova Solar, Walter Alvial S., Macarena Aro D. Ana Bonifetti D., Marilyn Hernández M., Claudio Rodríguez C. Problemas de salud mental en estudiantes de la Universidad de Concepción. Rev. Terapia Psicológica, 2007; 25(2): 105-112.

8. Beck, A. T., Rush, A. J., Shaw, B. F. \& Emery, G. Terapia Cognitiva de la Depresión. Bilbao. España, 2005; 20(2): 120-150)

9. Perales A, Sogi C, Morales R. Estudio comparativo de salud mental en estudiantes de medicina de dos universidades estatales peruanas. Rev. An Fac Med., 2003; 64(4): 239-246.

10. Amézquita M, González R, Zuluaga D. Prevalencia de la depresión, ansiedad y comportamiento suicida en la población estudiantil de pregrado de la Universidad de Caldas. Rev. Colomb. Psiquiatría, 2003; 32(4): 341-356.

11. Feldman, L., Goncalves, L., Chacón Puignau, G., Zaragoza, J., Bagés, N., \& De Paulo, J. (2008). Relaciones entre estrés académico, apoyo social, salud mental y rendimiento académico en estudiantes universitarios venezolanos. Universitas Psychologica. Scielo, 2008; 7(3): 739-751.

12. Francisco Romo Nava, Silvia A. Tafoya, Gerhard Heinze. Estudio comparativo sobre depresión y los factores asociados en alumnos del primer año de la Facultad de Medicina y del Internado. Revista Salud Ment, 2013; 36(5): 9-15.

13. Fisher $S$, Hood R. The stress of the transition to university: a longitudinal study of vulnerability to psychological disturbance and home-sickness. Rev. Online NCBI, 1996; 78(4): 41-42

14. Osornio-Castillo, L; Palomino-Garibay, L. Depresión en estudiantes universitarios. Archivos en Medicina Familiar, 2009; 11(1): 1-2.

15. Miranda Bastidas; Carlos Alberto; Gutierrez, Segura, Julio Cesar; Bernal, Buitrago, Ferney; Escobar, Carlos Andres. Prevalencia de depresión en estudiantes de medicina de la U. del Valle. Revista Colombiana de Psiquiatría, 2000; 29(3): 251-260.

16. Joffre Velázquez, Víctor Manuel, Martínez Perales Gerardo, Maldonado García Gerardo, Sánchez-Gutiérrez de Lara, Lucero. Depresión en estudiantes de medicina. Resultados de la aplicación del inventario de depresión de Beck en Alcmeon. Revista Argentina de Clínica Neuropsiquiátrica, 2007; 14(1): 86-93.

17. Czernik G, Giménez $S$, Mora $M$, Almirón L. Variables sociodemográficas y síntomas de depresión en estudiantes universitarios de Medicina de Corrientes, Argentina. Rev Arg Clin Neuropsiquiatrica, 2006; 13(2): 64-73.

18. Jou $H$, Fukada $H$. Stress and social support in mental and physical health of Chinese students in Japan. Psychol Rep, 1997; 81(3): 12-130.
19. Marty M., Matías Lavín G., Maximiliano Figueroa M., Demetrio Larraín de la C., Carlos Cruz M. Prevalencia de estrés en estudiantes del área de la salud de la Universidad de los Andes y su relación con enfermedades infecciosas. Scielo. Rev Chil NeuroPsiquiat, 2005; 43(1): 25-32

20. Stewarts $S M$, Betson C, Marshall I, Wong C M, Lee PW, Lam TH. Stress and vulnerability in medical students. Med Educ, 1995; 10(111): 136-292.

21. Medina-Mora, M.E., Borges, G., Lara, C., Benjet, C., Blanco, J.J., Fleiz B.C. Prevalencia de trastornos mentales y uso de servicios. Resultados de la encuesta nacional de epidemiología psiquiátrica en México. Rev. Salud Mental, 2003; 26(4): 1-16.

22. Gutiérrez Rodas, Javier Antonio; Montoya Vélez, Liliana Patricia; Toro Isaza, Beatriz Eugenia. Depresión en estudiantes universitarios y su asociación con el estrés académico. Revista CES Medicina, 2010; 24(1): 7-17.

23. Riveros $Q M$, Hernández $\vee H$, Rivera B J. Niveles de depresión y ansiedad en estudiantes universitarios de Lima Metropolitana. Revista IIPSI Facultad de Psicología, 2007; 10(1): 1-17.

24. Cantoral G, Méndez M V y Nazar A. Depresión en adolescentes: un análisis desde la perspectiva de género. 2005, Tesis disponible en: http//www.ecosur. rasecofronteraecofront21depresion.pdf.

25. Guillermo Valencia M. Riesgo de Depresión en Estudiantes del programa de Enfermería de la Universidad libre de Pereira. Revista Cultura Del Cuidado Enfermería. Colombia, 2010; 7(2): 14:26.

26. Terrones Saldívar Ma del Carmen. Prevalencia de depresión en estudiantes de algunas carreras del Centro de Ciencias de la Salud de la Universidad Autónoma de Aguascalientes. Revista LUX MÉDICA. Aguascalientes, 2014; 26(1): 15-19.

27. Sidana Surbhi, Kishore Jugal, Ghosh Vidya, Gulati Divyansh, Jiloha RC, Anand Tanu. Prevalence of depression in students of a medical college in New Delhi: A cross-sectional study. Nueva Dehli, India. Australasian Medical Journal, 2012; 5(5): 247-250.

28. Ferreira Furegato $A R$, Ferreira Santos JL, da Silva EC. La depresión entre estudiantes de enfermería relacionada a la autoestima, a la percepción de su salud y al interés por la salud mental. Rev. Latino-Am. Enfermagem, 2008; 16(2): 198-204.

29. Arrivillaga, M., Cortés, C., Goicochea, J., \& Lozano, T. Caracterización de la depresión en jóvenes universitarios.Colombia. Universitas Psicológica, 2003; 3(1): 17-26. 